\title{
Smith, Graham (2021): Can Democracy Safeguard the Future?
}

\author{
Cambridge: Polity Press. 160 Seiten. \$12.95
}

\author{
Julian Frinken
}

Angenommen: 6. Juli 2021 / Online publiziert: 2. August 2021

(C) Der/die Autor(en) 2021

Fragen der intergenerationellen Gerechtigkeit, wie sie sich etwa am drastischsten in der Bewältigung der Klimakrise stellen, werden zunehmend auch in der Demokratietheorie reflektiert. Aus deren Perspektive können sehr junge und noch nicht geborene Menschen nach dem Betroffenheitsprinzip als ein Teil des Demos gelten, dem jedoch keine demokratische Stimme zukommt. Zweifel an der demokratischen Fähigkeit langfristiger Problemlösung sind gerade mit Blick auf den Klimawandel inzwischen so weit verbreitet, dass sogar der Protest der Fridays for Future-Bewegung durch den Leitspruch ,follow the science“ eine technokratische Schlagseite bekommt.

Während die Frage, die Graham Smith mit seinem Buchtitel stellt, in diesem Licht als eine äußerst skeptische erscheinen muss, plädiert er zwischen den Buchdeckeln auf konzise Weise für eine ,Vertiefung“ der Demokratie. Dass hierin die Antwort auf die diagnostizierte ,demokratische Kurzsichtigkeit“ zu finden sei, erscheint auf den ersten Blick kontraintuitiv, wird jedoch dadurch aufgelöst, dass nicht Demokratie per se, sondern vor allem deren Formalisierung durch elektorale Verfahren als eine wesentliche Ursache für kurzsichtige Politik ausgemacht wird. Als weitere Faktoren werden im ersten Kapitel die Abwesenheit zukünftiger Generationen vom demokratischen Diskurs, der mächtige Einfluss kurzfristig orientierter Profitinteressen und die allgemeinere kapitalistische Gesellschaftsordnung identifiziert. Nachdem technokratische Lösungsansätze auf überzeugende Weise als Irrweg dargestellt werden, beinhaltet das zweite Kapitel eine Auseinandersetzung mit empirischen Erkenntnissen und Reformideen, welche die Möglichkeit der Verankerung weitsichtiger Politik in Legislative und Verfassung in den Blick nehmen. So wird deutlich, dass zentrale Herausforderungen darin bestehen, Legitimität für und gesellschaftliches Vertrauen

Julian Frinken $(\bowtie)$

Johannes Gutenberg-Universität Mainz, Mainz, Deutschland

E-Mail: frinken@politik.uni-mainz.de 
in Verfahren zu generieren, die die Interessen jener schützen, die diese selbst nicht artikulieren können. Da Gesellschaften auch in Zukunft nicht homogen sein werden, wäre es dabei verfehlt, künftigen Generationen bloß ein aggregiertes Kollektivinteresse $\mathrm{zu}$ unterstellen. Auch weitsichtige Politikentscheidungen müssten deshalb in einem Bewusstsein über ihre umverteilenden Effekte getroffen werden, sodass Ungleichheiten zwischen sozialen Gruppen zukünftiger Gesellschaften zumindest nicht gänzlich ausgeblendet werden. Sowohl legislative als auch verfassungsrechtliche Reformansätze seien in dieser Hinsicht jedoch aus dem Grund limitiert, dass gerade Parlamente und Verfassungsgerichtshöfe sozioökonomisch kaum divers zusammengesetzt seien. Im dritten Kapitel wendet sich Smith sogenannten ,offices for future generations“ (OFGs) als unabhängigen politischen Einrichtungen zu. Erfahrungen aus Israel und Ungarn zeigen, dass der Einfluss solcher Stellen stets dann drohe, beschnitten zu werden, wenn sie Parlament oder Regierung politisch herausfordern. Demokratietheoretisch stelle sich außerdem die stets wiederkehrende Frage, auf welcher Grundlage sie dazu legitimiert seien, fundierte Urteile über die Präferenzen künftiger Generationen zu treffen.

Die Antwort darauf sei in ihrer Kopplung an partizipative Verfahren zu finden. Denn eine breite Inklusion von gesellschaftlichen Perspektiven verhelfe, wie Smith im vierten Kapitel weiter ausführt, zur Legitimierung von kollektiven Entscheidungen und könne eine gerade für die unterschiedlichen Betroffenheiten von sozialen Gruppen sensible Annäherung an die Präferenzen zukünftiger Generationen leisten. Hier widmet er sich nun losbasierten und deliberativen Mini-Publics als für diese Zwecke geeigneten partizipativen Verfahren. Dabei charakterisiert Smith sowohl die diversifizierende und egalisierende Wirkung des Losverfahrens als auch die reflexive und rationalisierende Praxis der Deliberation als Eigenschaften, die weitsichtige Entscheidungsfindung befördern. Zentral ist an dieser Stelle das dem gesamten Werk zugrunde liegende Argument, wonach demokratische Kurzsichtigkeit nicht daher rühre, dass Bürger*innen an sich egoistische Maximierer*innen kurzfristigen Nutzens seien, sondern dass es wesentlich von der institutionellen Struktur der politischen Entscheidungsfindung abhänge, ob sich weitsichtige Erwägungen in Politik umsetzen ließen. Das überzeugt vor dem Hintergrund der Erkenntnis, dass Zeitpräferenzen stark von Kontext und Framing des betreffenden Gegenstandsbereichs abhängen und verweist somit auf die Bedeutung der Herstellung eines entsprechenden Rahmenwerks. Ob ein solches Projekt eher reformistisch angegangen werden solle, indem etwa Mini-Publics den OFGs zu Konsultationszwecken zur Seite gestellt werden, oder ob es eines radikaleren Ansatzes, etwa einen der Ersetzung von Teilen der Legislative durch losbasierte Kammern bedürfe, lässt Smith in seiner Diskussion offen. Doch er besteht darauf, dass eine Art von „empowerment“ geloster Foren, mindestens in Form eines Vetorechts, stattfinden müsse. Ansonsten könnten weitsichtige Impulse stets wieder durch die Kurzsichtigkeit bestehender Strukturen egalisiert werden. Neben dieser effektiven Bemächtigung leitet Smith aus seiner Diskussion schließlich die Kriterien von Unabhängigkeit, Diversität, Deliberation und Institutionalisierung als weitere zentrale Eckpfeiler des Designs einer weitsichtigen Demokratie ab.

Sich dem Problem der Unverfügbarkeit der Interessen zukünftiger Generationen durch eine möglichst breite Inklusion von zwangsweise gegenwärtigen Perspektiven 
zu nähern, überzeugt zunächst, doch die Institutionalisierung dieser Strategie durch Mini-Publics bringt eigene Legitimationsprobleme mit sich. Mini-Publics sind nicht allein als partizipative, sondern im Kern als repräsentative Foren zu begreifen, in denen die Teilnehmenden auf eine Weise als Repräsentant*innen der Außenstehenden agieren, von denen sie dazu jedoch keine direkte Mandatierung erhalten. Das Problem der fehlenden Autorisierung wie Smith durch den Hinweis darauf zu kontern, dass zumindest allen Bürger*innen die gleiche Chance darauf gegeben sei, in der Rolle der Repräsentierenden zu landen, greift zu kurz, da diese zudem nicht effektiv rechenschaftspflichtig gegenüber der Öffentlichkeit sind. Da im Grunde alle wichtigen politischen Fragen auf irgendeine Weise zukunftsrelevant sind, läuft diese Strategie auf eine umfassende und dabei problematische Übertragung von Entscheidungsmacht auf dazu nichtautorisierte Bürger*innen hinaus. Das verdeutlicht die Bedeutung der weiterführenden Frage, wie genau Wahlen auf der einen und die Losauswahl von Mini-Publics auf der anderen Seite bei der Herstellung legitimer Politiken verfahrenstechnisch ineinandergreifen können. Die von Smith angeführten Vetorechte könnten sich etwa unter noch näher zu bestimmenden Bedingungen als tragfähige Option erweisen, da sie die Rolle des Parlaments nicht grundsätzlich abwerten, jedoch dessen Responsivität hinsichtlich bestimmter diskursiv gefilterter Gründe zwangsweise erhöhen.

Smiths Beitrag zur Diskussion um die Weitsichtigkeit demokratischer Systeme stellt trotz seiner formalen Kürze eine breite und informative Überschau über empirische Erkenntnisse, erprobte Praktiken und existente Reformideen dar. Das damit verknüpfte Plädoyer für eine ,vertiefte“ Demokratie richtet sich auch dank der eingängigen Sprache an ein breites Publikum, welches diesem Werk zu wünschen ist. Denn Smiths Ideen verdienen es, kritisch weitergedacht und konkretisiert zu werden.

Funding Open Access funding enabled and organized by Projekt DEAL.

Open Access Dieser Artikel wird unter der Creative Commons Namensnennung 4.0 International Lizenz veröffentlicht, welche die Nutzung, Vervielfältigung, Bearbeitung, Verbreitung und Wiedergabe in jeglichem Medium und Format erlaubt, sofern Sie den/die ursprünglichen Autor(en) und die Quelle ordnungsgemäß nennen, einen Link zur Creative Commons Lizenz beifügen und angeben, ob Änderungen vorgenommen wurden.

Die in diesem Artikel enthaltenen Bilder und sonstiges Drittmaterial unterliegen ebenfalls der genannten Creative Commons Lizenz, sofern sich aus der Abbildungslegende nichts anderes ergibt. Sofern das betreffende Material nicht unter der genannten Creative Commons Lizenz steht und die betreffende Handlung nicht nach gesetzlichen Vorschriften erlaubt ist, ist für die oben aufgeführten Weiterverwendungen des Materials die Einwilligung des jeweiligen Rechteinhabers einzuholen.

Weitere Details zur Lizenz entnehmen Sie bitte der Lizenzinformation auf http://creativecommons.org/ licenses/by/4.0/deed.de. 